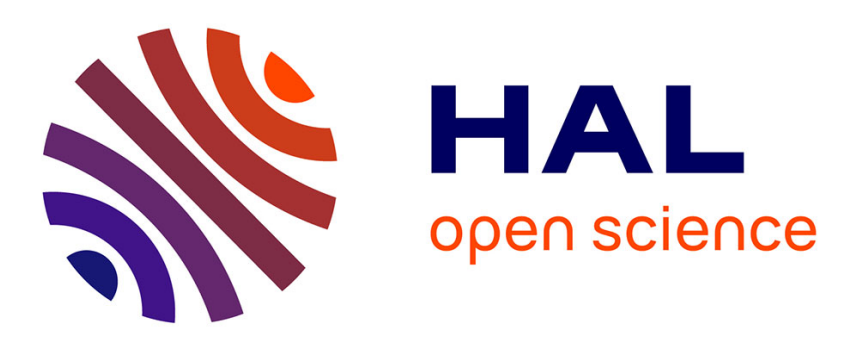

\title{
L'intuition catégoriale de la relation : le renversement husserlien
}

\author{
Emmanuel Housset
}

\section{To cite this version:}

Emmanuel Housset. L'intuition catégoriale de la relation : le renversement husserlien. Les études philosophiques, 2017, Descartes et la phénoménologie en Pologne et en Europe centrale, 2 (172), pp.289-306. 10.3917/leph.172.0289 . hal-02147437

\section{HAL Id: hal-02147437 \\ https://hal-normandie-univ.archives-ouvertes.fr/hal-02147437}

Submitted on 4 Jun 2019

HAL is a multi-disciplinary open access archive for the deposit and dissemination of scientific research documents, whether they are published or not. The documents may come from teaching and research institutions in France or abroad, or from public or private research centers.
L'archive ouverte pluridisciplinaire HAL, est destinée au dépôt et à la diffusion de documents scientifiques de niveau recherche, publiés ou non, émanant des établissements d'enseignement et de recherche français ou étrangers, des laboratoires publics ou privés. 
Emmanuel HOUSSET

Université de Caen Normandie Equipe

Identité et Subjectivité (EA 2129)

\section{L'intuition catégoriale de la relation : le renversement husserlien}

Le terme de relation a pris aujourd'hui une place tout à fait prépondérante dans la philosophie du sujet comme dans son dépassement. En effet, si, par exemple avec Kierkegaard ${ }^{1}$ ou avec les philosophies du dialogue (Buber, Rosenzweig, Löwith) ou encore avec le personnalisme (Gabriel Marcel, Nédoncelle), la relation se trouve décrite en termes d'être (le moi, selon des figures très diverses, n'a pas des relations, il est une relation), de co-esse ${ }^{2}$, la catégorie de la relation semble devenir la première des catégories, mais en même temps, en prenant cette première place dans une philosophie de l'existence, elle cesse aussi sans doute d'être une catégorie, une détermination de l'être, pour devenir la vie même de l'être dans une co-naissance, voire l'ouvert à l'au-delà de l'être dans l'effraction de la transcendance. C'est, ce qui a lieu avec la philosophie de Levinas qui met en évidence l'essence relationnelle (et non pas intersubjective) du sens liée à la compréhension de l'être non plus comme substantif, mais comme verbe. Il serait également possible d'envisager ce que Merleau-Ponty entend par chair dans Le visible et l'invisible, ce qui selon lui n'a de nom dans aucune philosophie, et qui est ce lien du moi et des choses, ce chiasme du moi et d'autrui, cet Ineinander qu'il tente de dire avec le terme de réversibilité et qui relève du préthéorique. Le lien devient alors le véritable fondement, le fondement d'avant le fondement, qui cependant échappe toujours au pur regard de l'essence. Par rapport à de telles ouvertures vers une relation originaire, qui se donne tout en se réservant (mais qui est considérée soit comme préthéorique soit comme éthique, ce qui est radicalement différent), Husserl est sans doute l'un des derniers à considérer encore la relation comme une catégorie et il est possible d'avancer qu'il propose une analyse phénoménologique de la relation dans laquelle la relation devrait tomber encore sous la lumière de l'évidence. Si on part de l'idée que le propre de la relation est de signifier qu'un prédicat n'est intelligible que dans le rapport d'un étant à un autre étant, il faut reconnaître que la phénoménologie met en

\footnotetext{
${ }^{1}$ Dans cette philosophie de l'existence le moi est un rapport qui se rapporte à lui-même tout en étant posé par un autre. Voir le début de La maladie à la mort.

${ }^{2}$ Voir ce qu'en dit Levinas dans Hors sujet, Fata morgana, 1987, p. 38.
} 
lumière qu'un étant ne se donne jamais seul et qu'il se donne au contraire toujours en rapport à d'autres étants. Dès lors, la phénoménologie est conduite à donner une place essentielle à la relation dans la vision des essences. Dans la mesure où tout étant est corrélatif des autres étants en se donnant sur l'horizon du monde, il y a bien non seulement une intuition de la relation, mais également une extension de la relation au mode de donnée de tout étant. Telle est la thèse essentielle par rapport à toute conception constructiviste de la connaissance : la relation ne se trouve pas déduite à partir de l'observation des étants et c'est bien elle qui se donne, même si une telle «donnée » peut sembler énigmatique. Il est alors possible de dire que voir, c'est toujours voir une relation, même si Husserl distingue les perceptions simples et les perceptions catégoriales. Au moins est-il sans doute possible d'avancer que même dans une perception simple il y a une dimension catégoriale. On s'éloigne alors des apories traditionnelles : rien ne se donnant de manière isolée (même le moi, ou Dieu), il n'y a plus à se demander ce qu'est un être consistant dans la relation à autre chose. L'alternative classique depuis la philosophie antique selon laquelle soit la relation est une altérité absolue, existant par soi (Platon), soit elle n'est qu'une propriété de la quantité, qui est elle-même une propriété des substances (Aristote) ${ }^{3}$, est alors en partie dépassée, même si un auteur comme Levinas renvoie explicitement à Platon afin de défendre la thèse qu'il y a au sein même de l'être la possibilité de l'altérité. Pour le dire autrement, le retour aux choses mêmes revendiqué par la phénoménologie permet d'avancer qu'il ne peut pas y avoir uniquement la substance et que la relation elle-même relève de l'être, et c'est pourquoi elle est une altérité, comme Husserl lui-même pourra l'envisager dans ses analyses sur l'intersubjectivité. En effet, Husserl ne cesse de l'élucider dans la cinquième des Méditations cartésiennes, toute personne est l'une des personnes et pourtant l'autre subjectivité se donne à partir de moi comme une altérité. Dire qu'autrui se donne comme un analogon de moi-même, c'est à la fois penser l'empathie et reconnaître qu'il n'y a pas d'accès direct à l'autre subjectivité. Il s'agit donc de montrer que la relation ne peut pas toujours être considérée comme un simple accident de la substance et que, par exemple, la paternité n'est pas ce que l'on pourrait perdre sans cesser d'être soi. Ainsi, devenir père ou cesser de l'être ne sont pas des accidents de la personnalité qui viendraient simplement s'ajouter à une substance déjà là, dans la mesure où ici la relation est la personnalité elle-même. Dès lors, ce ne sont pas seulement le père, le fils ou l'ami qui sont des relatifs, mais l'être-homme lui-même. Selon cette nouvelle perspective, la relation ne peut pas être considérée comme ce qui viendrait s'ajouter à une physis, en tant que l'une de ses ramifications possibles, puisque dans l'exemple considéré il ne s'agit plus de concevoir la paternité, et tout aussi bien la fraternité ou la maternité, comme ce qui viendrait simplement s'ajouter à l'essence de l'homme. Notamment la phénoménologie de Husserl, en montrant que l'histoire ne peut être réduite à un ensemble de faits contingents advenant à un sujet anhistorique et qu'au contraire l'historicité est la subjectivité elle-même dans son autoconstitution ${ }^{4}$, met également en lumière, par cette compréhension de l'être comme histoire, la nécessité de dépasser l'opposition de la substance et des accidents. Selon cette perspective, la relation ne saurait être comprise comme une pure réalité nominale, qui reconduirait à notre capacité de synthèse et donc de jugement. En effet, l'opposition brutale de la substance et des accidents se trouve dépassée par l'idée que la synthèse devient la donnée même de l'expérience : "être le père de », comme « être à la droite de », n'apparaissent pas à la faveur d'un jugement ayant lieu après l'expérience et doivent être considérés comme des

\footnotetext{
${ }^{3}$ Voir Crubellier Michel, « Domestiquer l'excès de l'être. La catégorie des relatifs entre Platon et Aristote », dans revue Quaestio 13/2013, dir. Vincent Carraud et Pasquale Porro, p. 3-15.

${ }^{4}$ Sur cette question voir Pradelle Dominique, Généalogie de la raison, Paris, PUF, 2013.
} 
« données » de l'expérience, comme relevant de la phénoménalité. Pour parler avec Husserl, il s'agit là d'une «nuance » qui porte en elle le sens même de la réduction phénoménologique, dans la mesure où la relation ne se donne pas comme extrinsèque, mais comme intrinsèque : le fils demeure fils même après la mort du père et telle ville demeure au sud de telle autre y compris dans l'hypothèse de la destruction de cette dernière. Pour prendre un exemple plus présent dans les travaux de Husserl, l'Europe demeure relative aux autres traditions, même si elle vient à mourir. Il est donc possible d'avancer d'une façon plus générale que les relations spatiales, temporelles et spirituelles ne sont pas extérieures à l'être et, d'une certaine façon, dans le monde comme monde tout est corrélatif. Bien évidemment, au-delà de Husserl, dans la philosophie de Heidegger, cette thèse devient indissociable de l'idée d'une appartenance réciproque de l'homme et du monde et, dans cette nouvelle compréhension du monde, il n'est plus question pour la connaissance de tenter de remonter du fait à l'essence, du constitué au constituant, comme on remonte à un non-relatif. Dans ce refus de considérer l'être en tant que substance accompagnée d'accidents relatifs, il est également possible d'accéder à la coappartenance des choses qui demeurait masquée par l'opposition de l'être absolu et des relatifs. Heidegger, dans Acheminement vers la parole, et donc cette fois au-delà de tout reste de kantisme, décrit très précisément en quoi le poète est celui qui appelle les choses à se manifester dans leur coappartenance. Cette fois «être» signifie «venir en présence » et Heidegger montre qu'une chose ne vient jamais seule en présence ; de cette façon la présence ne se trouve pas figée en constance. Dans le poème de Trakl commenté par Heidegger, Un soir d'hiver, la maison, la neige, etc. ne sont pas des choses constantes et le retour aux choses mêmes doit conduire à comprendre comment elles séjournent ensemble. La chose n'est chose que dans ce rassemblement.

Revenir au fondement, ce n'est pas nécessairement accéder à de l'absolu, à du nonrelatif, et il est possible de se demander si ce n'est pas cela qui permet de rompre ultimement avec une conception soit empiriste, soit idéaliste, de la relation. Quoi qu'il en soit, la phénoménologie dès ses débuts modifie la compréhension de la relation catégoriale en ne concevant plus la relation à partir de la distinction du sujet et des accidents, car une telle distinction contrevient à la phénoménalité. Etre-avec-autrui, être-au-monde, « être au sud de », ne sont pas des accidents, et cela induit une nouvelle intelligence de la substantialité avec l'idée d'une relation essentielle. Encore une fois, « être plus grand que » n'est pas une détermination extérieure à la chose et qui ne viendrait pas d'elle-même, mais d'un jugement extérieur, d'une comparaison. Il s'agit au contraire d'une détermination qui se trouve donnée avec la chose même et il est possible de défendre la thèse selon laquelle, même pour les simples choses, la relation n'est pas l'advenue d'une altérité qui ne proviendrait pas de la chose même et qui la déterminerait sans la modifier. En conséquence, ce n'est pas seulement tel élément d'une machine, ou tel organe d'un corps, qui n'est pas donné d'une manière isolée, mais toute chose selon son apparaître. Rien n'est par soi seul et une telle thèse que l'on trouve dans les dernières œuvres de Heidegger et qui a des racines nietzschéennes suppose un renversement complet de l'ontologie traditionnelle, dans la mesure où elle suppose que l'identité ne soit plus un trait de l'être et que tout être soit un devenir. Bien évidemment jamais Husserl n'ira aussi loin et jamais il ne pensera la relation au-delà du théorique ; ainsi jamais la réduction phénoménologique ne conduira à remettre en cause le principe d'identité, et il faut attendre les dernières analyses de Heidegger pour que la relation n'ait plus sa signification métaphysique, pour qu'elle ne soit plus comprise comme une catégorie, afin d'être vue en tant qu'acte de différenciation que Heidegger nomme Dif-férence. Toute la philosophie heideggérienne de l'Ereignis, qu'il n'est 
pas possible d'exposer ici, montre comment les choses se différencient entre elles au lieu de se distinguer par rapport à une essence commune. La phénoménologie s'accomplit, et pour certains se perd, dans cet accès à une différence qui se trouve dans la phénoménalité elle-même. Aller au-delà de la différence ontologique de l'être et de l'étant afin de décrire l'autodifférenciation de l'être, c'est enfin échapper à cette distinction de la substance et des accidents qui ne vient pas des choses mêmes, c'est cesser véritablement d'intellectualiser les phénomènes. La phénoménologie a donc pu donner à voir que l'altérité des choses elles-mêmes ne doit plus être seulement comprise de manière catégoriale. Néanmoins, cette relation originaire, antérieure aux catégories, n'est-elle pas ce qui échappe, comme toute origine, au regard ? Cette brève mise en perspective historique de la question décisive de la relation va permettre de mieux comprendre la place propre de la phénoménologie de Husserl, qui là encore est bien un tournant dans la philosophie en ce qu'elle ouvre des perspectives qui vont bien audelà d'elle-même et donc une nouvelle histoire du concept de relation.

Le projet du présent travail n'est cependant pas d'exposer toutes les analyses de Husserl sur la relation, ni même d'élucider la naissance de la compréhension husserlienne de la relation ${ }^{5}$ et sur la façon dont il veut dépasser toute conception empiriste ou idéaliste, mais il s'agit de montrer avec Husserl, en suivant les analyses de la sixième des Recherches logiques, que la relation n'est pas seulement l'occasion d'assembler deux idées afin de les appréhender ensemble, mais qu'elle est une certaine forme intuitive du « et », de «l'être-ensemble ». Il y a ainsi avec Husserl un renversement de toute conception intellectualiste de la relation et il s'agit de montrer également qu'au-delà de ses difficultés et des limites qu'elle se pose, cette nouvelle intelligence de la relation va s'entendre à des champs très différents (le passé, autrui, la communauté des personnes) et surtout qu'elle va rendre possible son propre dépassement avec la thèse que la relation doit être pensée au-delà de l'ontologie comme un événement et non plus comme un état. Tel est le sujet d'étonnement : comment se fait-il qu'au moment où la relation semble devenir la plus importante des catégories dans une ontologie phénoménologique on assiste à la fin de l'ontologie avec l'idée d'une relation à l'infini comme source du sens ?

\section{La relation n'existe pas que dans 1'appréhension : Husserl et Kant}

Toutes les analyses de Husserl sur la relation sont aussi une confrontation permanente au tournant kantien et donc avec cette thèse kantienne selon laquelle la relation ne serait qu'une structure subjective qui se rapporte notamment à la possibilité de la physique mathématique. Dans la Critique de la raison pure la relation est le troisième titre de la table des catégories et comporte inhérence et subsistance ; causalité et dépendance ; communauté ou action réciproque entre l'agent et le patient ${ }^{6}$. Il s'agit de montrer que dans le monde phénoménal il n'y a de connaissance que des relations : dans l'intuition externe il y a une saisie des rapports des objets dans l'espace et le temps, mais cette connaissance porte uniquement sur le rapport de l'objet au sujet dans sa représentation et non sur la chose en soi. Il en va de même pour l'intuition interne et les rapports de succession et de simultanéité. D'une façon générale, l'espace et le temps étant de purs rapports formels, la relation reconduit à la manière dont l'objet est représenté. De ce

\footnotetext{
${ }^{5}$ Voir sur ce point l'étude de Vincent Gérard, «La théorie de la relation et la genèse de l'ontologie formelle », dans Lectures de Husserl, Paris, Ellipses, 2010, p. 39-76.

${ }^{6}$ Pour la Critique de la raison pure nous renvoyons à la traduction par Alexandre J.-L. Delamarre et François Marty, dans Euvres philosophiques I, Bibliothèque de la Pléiade, Paris, Gallimard, 1980. Pour la table des catégories (A 80/B 106) p. 834.
} 
point de vue, la relation causale devient le modèle pour penser la relation de la substance à l'accident et la relation du tout à la partie. Certes ces relations sont constitutives de l'objectivité, mais elles proviennent de l'application des règles de la synthèse par l'entendement, qui ainsi transforme le donné en objet construit. En conséquence, si la relation devient avec Kant une structure de la subjectivité, elle n'est pas pour autant saisie intuitivement et bien au contraire elle ne peut être déterminée que selon une méthode régressive à partir de la table des jugements.

Afin d'élucider la relation, Husserl va s'éloigner de l'empirisme comme de l'idéalisme kantien et, dans son refus de l'anthropologie, de toute conception subjective de l'apriori, en mettant fin à la dissociation de la vision sensible et de la vision catégoriale ${ }^{7}$. Un tel éloignement s'explique peut-être par le fait que le concept de relation, si important dans l'ontologie médiévale telle qu'elle culmine avec saint Thomas d'Aquin ${ }^{8}$, perd sans doute de sa force quand il se déploie dans le cadre d'une doctrine du jugement, dans la mesure où la catégorie de relation est plus «simple » à concevoir comme catégorie de l'être que comme catégorie du jugement. En effet, la relation semble se penser plus difficilement dans une métaphysique de la subjectivité, car non seulement elle demeure extrinsèque aux choses, mais en outre on peut être conduit à confondre «être relatif à » et « être en relation avec». Or « être à la droite de » ou bien «être esclave de » désignent le relatif au sens aristotélicien, c'est-à-dire le prédicat d'un sujet relatif à autre chose, alors que « être en relation avec » renvoie à un prédicat qui se dit de deux sujets liés entre eux, tel qu'être des opposés l'un par rapport à l'autre pour les nombres. D'une certaine manière, Husserl va vouloir rendre sa force à la catégorie de relation en introduisant un renversement complet de perspective, puisque cette fois l'être va se trouver phénoménalement présent dans cette catégorie de relation, qui ne pourra plus être considérée comme un rejeton de l'être ou comme un simple être de raison. Comme le dit Heidegger, « Husserl a libéré l'être de sa fixation dans le jugement ${ }^{9}$ », et c'est cela qui permet à la relation d'apparaître dans toute sa consistance ontologique. Or cela résulte de la méthode intuitive à l'œuvre dès les Recherches logiques : l'intuition prise au sens élargi, c'est-à-dire sans la limiter à l'intuition sensible, est la seule source de droit pour la connaissance, notamment pour dégager les lois de la logique pure. De ce fait, le statut de la relation s'en trouve profondément modifié, dans la mesure où l'intuition contient bien plus que la manière dont le sujet est affecté par l'objet. En effet, si l'intuition sensible est le remplissement d'une visée singulière, elle est également le fondement d'une saisie de l'essence et de la relation, qui ne sont plus considérées comme de simples constructions psychiques. Selon cette nouvelle perspective, non seulement l'universel est visible, mais le relatif se donne lui-même dans l'expérience ; plus précisément, la relation appartient à l'objet apparaissant comme tel, car la perception n'est pas purement passive ; elle n'est pas une sensation accompagnée de conscience et elle est au contraire un acte donnant à voir.

Husserl, encore une fois, s'évertue à distinguer « être relatif à » et «être en relation avec », notamment quand dans la sixième des Recherches logiques il met en lumière que l'évidence peut porter sur les pures relations, comme l'impossibilité de saisir la couleur sans

\footnotetext{
${ }^{7}$ Il s'agit d'un point particulièrement souligné par Heidegger dans le séminaire de Zähringen : « Le tour de force de Husserl a justement consisté dans cette mise en présence de l'être, phénoménalement présent dans la catégorie », Seminare, Gesamtausgabe, tome 15, Francfort, Klostermann, 1986, p. 378 ; trad. Questions IV, Paris, Gallimard, 1976, p. 315.

${ }^{8}$ Voir sur ces questions historiques l'ensemble du volume déjà cité : Quaestio 13/2013, dir. Vincent Carraud et Pasquale Porro.

${ }^{9}$ Heidegger, Seminare, p. 377 ; trad., p. 315.
} 
l'étendue. De même dans l'acte de nommer mon encrier, il y a une relation essentielle entre l'acte d'intuition et l'acte de nomination, entre l'acte sensible et l'acte catégorial :

Ce n'est donc pas le mot et l'encrier qui entrent en relation, mais les vécus d'actes que nous venons de décrire, dans lesquels ils apparaissent, alors qu'ils ne sont absolument rien «en» eux. Comment cela a-t-il donc lieu ? Qu'est-ce qui unifie ces actes ? La réponse paraît claire. Cette relation, en tant que dénominative, est médiatisée par des actes qui ne sont pas seulement ceux du signifier, mais aussi ceux du connaître et à coup sûr ce sont, en ce cas, des actes de classification $^{10}$.

Au-delà d'une relation entre le mot et l'encrier, la phénoménologie dévoile une relation entre l'acte de signifier (la classification) et l'acte de perception. Ensuite, c'est le travail de la pensée à partir de l'expérience qui transforme une relation qui peut être d'abord extrinsèque en une relation intrinsèque. Le projet de Husserl est avant tout de montrer que la perception est la saisie d'une unité entre le mot encrier et la donnée intuitive de l'encrier ; cette unité ne résulte pas d'une comparaison, mais est la perception elle-même. Pour prendre un autre exemple que ceux de Husserl, dans la parole biblique Ecce homo, il s'agit bien de voir l'Homme dans l'homme et non d'effectuer une confrontation de contenus. Cela n'est pas mis en péril par le fait que, bien évidemment, l'expérience peut être également non concordante et que dans ce cas le monde donné ne correspond pas au monde visé et qu'il y a alors conflit entre l'intuition et la signification.

\section{L'intuition de la relation}

Toute l'analyse des relations possibles entre intuition et signification (perception, imagination, souvenir) vise à montrer qu'il est impossible de limiter l'intuition à l'intuition sensible et que la signification et l'intuition ne peuvent être comprises séparément : toute la question est bien celle de leur relation. Pour élucider cela, il convient de revenir à l'idée que le rapport de la sensibilité et de l'entendement est compris d'une manière tout à fait nouvelle par Husserl, dans la mesure où la synthèse a lieu dès la sensibilité et qu'en conséquence, pour que l'être se donne, il doit y avoir une intuition catégoriale. Telle est la percée de Husserl qui vient briser les digues de la représentation. En effet, en faisant de l'objet, et non du sujet, le fil conducteur de la connaissance, Husserl peut mettre en lumière que ce qui est donné dans l'intuition catégoriale « est». Ainsi la relation comme catégorie devient un « objet» au sens propre du terme, puisque dans une signification très élargie de l'être, tout ce qui vérifie une visée « est ». En conséquence, en suivant l'exemple pris par Husserl « Je vois que ce papier est blanc $»^{11}$, voir, c'est également voir un acte de liaison, un acte de mise en relation. Autrement dit, sur la base de la perception sensible, il y a aussi une intuition de la relation. Notamment l'expérience peut porter sur des relations entre des unités idéales, comme pour les figures géométriques. Husserl précise alors bien avec l'exemple du papier blanc que « l'être n'est rien dans l'objet ${ }^{12}$ », qu'il n'est pas un donné perceptif de l'objet; néanmoins s'il n'est pas un caractère de l'objet, il n'est pas rien non plus dans l'objet. Ainsi l'être n'est rien de perceptible et pourtant il est donné quand je dis « ce papier est blanc ». Il y a donc bien intuition catégoriale et ce qui est vrai de l'être est vrai de toutes les catégories. Cela conduit à reconnaître que la

\footnotetext{
${ }^{10}$ Husserl, Recherches logiques, Recherche VI § 6, Husserliana Tome XIX/2, édité par Ursula Panzer, La Hague/Boston/Lancaster, Nijhoff, 1984, p. 559 ; trad. Hubert Elie, Arion L. Kelkel et René Schérer, Paris, PUF, 1974, p. 39.

${ }^{11}$ Recherches logiques VI, § 40, p. 659 ; trad. p. 163.

${ }^{12}$ Recherches logiques VI, § 43, p. 666 ; trad. p. 169.
} 
relation elle-même ne naît pas d'une réflexion sur les actes psychiques et qu'elle est, au contraire, donnée. Je ne vois pas A, puis B, mais A plus grand que B. Le renversement propre aux Recherches logiques consiste à mettre en évidence que la relation est bien une donnée de l'expérience, que l'objet est là en chair et en os devant nos yeux, y compris dans sa relation. La catégorie de la relation n'est pas ce qui permet de penser l'objet, mais elle est bien ce qui le donne. Comme Husserl le dira dans le $\S 44$ des Méditations cartésiennes, voir l'autre, c'est voir ce qui renvoie, de façon constitutive, à moi-même ${ }^{13}$. De manière plus proche des Recherches logiques, les études sur le temps de 1905 montre qu'il y a intuition du maintenant, intuition de l'instant passé dans la rétention et intuition de leur relation. L'intuition de la succession suppose de saisir ensemble l'être passé et l'être présent ; de même la saisie du présent est corrélative d'une attente intuitive et il y a donc bien également une intuition d'une relation entre le présent et le futur. Contre Brentano Husserl met en lumière que la relation n'est pas une construction de l'imagination, mais une intuition et dès lors il peut affirmer : «L'évidence de la conscience temporelle : aussi loin s'étend la continuité ininterrompue, aussi loin s'étend l'évidence ${ }^{14} \gg$. Sans multiplier les renvois à des champs d'analyse de Husserl, il est possible de dire que l'élucidation phénoménologique de la transcendance du passé et de la transcendance de l'autre est la conséquence directe de la désubjectivisation de la relation. En conséquence, la relation est maintenant un «objet» de perception, sans être pour autant simplement donnée par la sensibilité, et elle cesse d'être comprise comme une catégorie de l'entendement qui viendrait assurer l'unité du divers de l'intuition.

Tout cela permet d'avancer que la catégorie de la relation se donne elle-même selon un remplissement non-sensible, même si elle se fonde sur l'intuition sensible de substrats. Comme le montre Husserl, l'ensemble mathématique est une forme catégoriale indépendante de la perception d'une pluralité de choses sensibles, qui est bien la donnée d'une liaison entre A, B, C... Ici peut être saisie de manière exemplaire l'idée que la saisie de l'ensemble ne vient pas après coup par une réflexion sur chacun des éléments et que cet ensemble est directement vu lui-même : il y a une intuition de l'être-ensemble des objets de cette pluralité. Contre Locke, Husserl peut alors établir que la catégorie de la relation ne vient pas de l'expérience interne, mais se trouve dans la donnée même des choses. En établissant ainsi que la catégorie de la relation ne vient pas d'une réflexion sur les jugements, mais des jugements eux-mêmes, Husserl peut avancer la thèse que la relation est une catégorie de l'être, mais dans un sens très différent de celui d'Aristote. Certes la relation a son lieu dans le jugement, mais elle n'est pas issue du jugement; elle vient de la donnée elle-même et cela même si cette donnée demeure problématique dans la mesure où l'être de la relation semble présupposer l'être de ce qui fonde la relation : «La forme, le devenir-intuitif dans l'évidence la plus générale, est pourtant fondée par les soubassements! Par conséquent, l' "être" de la relation présuppose l'être des fondements : l'être donné de la relation celui des fondements. Voire $!^{15}{ }^{15}$.

La perception de la relation suppose, comme toute perception catégoriale, une perception sensible, même si la relation est bien un objet qui se donne en personne et non une

\footnotetext{
${ }^{13} \mathrm{Il}$ est possible aussi de penser à toute l'ontologie de la socialité développée par Husserl et à son souci de mettre au jour ici également un apriori. Voir l'étude très complète de Laurent Perreau, Le monde social selon Husserl, Springer, 2013.

${ }^{14}$ Husserl, Husserliana X, Nijhoff, 1966, p. 169-170, trad. J.-F. Pestureau, Sur la phénoménologie de la conscience intime du temps, Millon, 2003, p. 84. Sur cette question voir les analyses très précises de Jean-François Lavigne, Husserl et la naissance de la phénoménologie, Paris, PUF, 2005, notamment p. 483 et sv.

${ }^{15}$ Husserliana X, op. cit., p. 190 note 1, trad. p. 100 note 45.
} 
simple production psychique. Encore une fois, la phénoménologie se veut le seul véritable empirisme, selon lequel la relation n'est pas dans l'esprit de l'homme, mais bien dans les choses mêmes. De cette façon elle n'est pas une simple forme vide en attente de contenu, mais elle résulte d'une donation non sensible, et en cela elle est l'être de l'objet, sa raison. Ainsi l'intuition catégoriale, par exemple dans la relation des parties au tout, est un autre regard sur le même objet, qui donne à voir ce qui le structure et quelles sont ses relations constitutives :

Mais nous pouvons aussi appréhender le même objet d'une manière qui l'explicite : dans des actes d'articulation, nous « faisons ressortir » les parties ; dans des actes relationnels, nous mettons ces parties ainsi dégagées en relation soit les unes avec les autres, soit avec le tout. Et c'est seulement grâce à ces nouveaux modes d'appréhension que les membres ainsi reliés et mis en relation acquièrent le caractère de « parties » ou de « touts ${ }^{16}$.

Le rapport du tout aux parties dit la nature idéale de l'objet dans un rapport de fondement, puisque « l'objet n'apparaît pas avec de nouvelles déterminations réelles, il est bien là, bien le même, mais selon un mode nouveau ${ }^{17}$ ». En effet, l'objet, qui était considéré pour lui-même dans la perception sensible, devient maintenant membre d'une relation, il entre dans un acte relationnel avec un sens idéal, tout en demeurant objet perçu. Or une telle relation de l'objet à l'apriori n'apparaissait pas avant le regard eidétique. Pour reprendre l'exemple de Husserl, percevoir A plus rouge que B, c'est effectivement voir une relation qui se trouve dans les choses elles-mêmes. De cette façon Husserl ne limite pas sa réflexion sur la relation au rapport du tout et des parties, mais considère également les relations externes entre les formes sensibles comme «être à la droite de » ou «A est plus clair que B ». Là encore il s'agit de distinguer deux regards : d'abord celui de l'intuition sensible dans laquelle A apparaît comme plus clair que $\mathrm{B}$, et là c'est la totalité ou l'être-ensemble qui est vu, donc une totalité qui est autre chose que la somme des parties. Ensuite le regard peut viser la relation elle-même ; il s'agit de voir la catégorie même de la relation ${ }^{18}$. La conversion du regard consiste donc à devenir attentif à la relation catégoriale et ces relations entre les objets constituent des objectivités d'ordre supérieur.

\section{La place privilégiée de la catégorie de relation}

Il est clair maintenant que le projet de Husserl est de rompre totalement avec la conception kantienne des catégories selon laquelle «elles sont des concepts d'un objet en général, par lequel l'intuition de cet objet est considérée comme déterminée à l'égard d'une des fonctions logiques du jugement ${ }^{19} »$. Selon L'analytique transcendantale, la catégorie est une fonction de détermination, elle donne l'unité, et la diversité des catégories ne vient qu'exprimer les différentes modalités de cette détermination. C'est bien pour cela que la diversité des catégories correspond à la table des jugements. Or si «les catégories sont des concepts qui prescrivent a priori des lois aux phénomènes et par conséquent à la nature, comme l'ensemble de tous les phénomène ${ }^{20}{ }^{\prime}$, elles ne sont pas issues de la nature et ne font qu'assumer a priori la liaison du divers de la nature. Ainsi Kant développe déjà particulièrement les catégories de la relation : inhérence et subsistance, causalité et dépendance, communauté (action réciproque

\footnotetext{
${ }^{16}$ Recherches logiques VI, § 48, p. 681 ; trad. p. 186.

${ }^{17}$ Recherches logiques VI, § 49, p. 686 ; trad. p. 191.

18 Voir Recherches logiques VI, §50.

${ }^{19}$ Critique de la raison pure (A95/B128), p. 850.

${ }^{20}$ Critique de la raison pure (B163), p. 875.
} 
de l'agent et du patient). En fin de compte, dans le monde phénoménal il n'y a que des relations et celles-ci tiennent à la relation au sujet qui connaît, et c'est pourquoi elles ne sont pas constitutives des choses-mêmes ${ }^{21}$. Sans pouvoir entrer ici pleinement dans la philosophie de Kant, on ne peut que constater cette importance de la catégorie de relation, par exemple de l'analogie comprise comme ressemblance parfaite de deux rapports entre des choses dissemblables. Bien évidemment il est possible de renvoyer également aux analogies de l'expérience, qui ne sont pas constitutives mais régulatrices. De même, la permanence de la substance, la succession dans le temps selon la causalité, la simultanéité selon la loi de l'action réciproque, permettent de se représenter l'unité de la nature. Ainsi la relation ne vient pas de l'expérience sensible et elle n'est pas non plus une catégorie innée, mais elle est bien produite par l'activité rationnelle. Comme cela fut souvent mis en lumière ${ }^{22}$, Kant reprend la qualité, la quantité et la relation de la table des catégories d'Aristote, et du coup il ne s'agit pas du tout de catégories au sens propre, mais plutôt de noms-titres pour plusieurs catégories. On parle désormais « des » catégories de la relation (Substance, causalité et réciprocité), qui sont bien sûr décisives pour la science. En effet, la substance se trouve dans la catégorie de la relation sans contenir une relation, mais parce que la permanence et le changement dans les représentations présupposent une relation ${ }^{23}$. Encore une fois, selon la perspective critique, ces concepts de relation ne sont pas des concepts des choses en elles-mêmes, qui demeurent inconnaissables, et ils ne sont pas plus des concepts empiriques, car ils sont nécessaires. En conséquence, la substance, la causalité et la réciprocité sont bien des concepts dérivés de l'entendement. D'une façon générale, la relation relève de la logique transcendantale, c'est-àdire à l'usage de la catégorie dans la construction de la connaissance de l'objet. Elle doit être comprise comme un principe de la possibilité de l'expérience, c'est-à-dire ce dans quoi un donné doit s'inscrire pour que l'expérience de quelque chose soit possible.

Husserl ouvre donc une toute nouvelle perspective avec l'intuition catégoriale de la relation, qui n'est justement pas cette fois une synthèse extérieure à la donnée, mais est la donnée elle-même :

Si nous parlons d'un acte qui réunit ces perceptions, et non d'une connexion quelconque ni même d'une association de ces perceptions dans la conscience, cela tient naturellement à ce qu'ici c'est une relation intentionnelle unitaire qui nous est donnée, et qu'il lui correspond un objet unitaire, lequel ne peut se constituer que dans cette connexion établie par un acte, tout comme un état de chose ne peut se constituer que dans l'acte de lier qui met en relation des représentations ${ }^{24}$.

Cela signifie par exemple que les chaises d'une salle de cours peuvent également être visées selon leur être-ensemble, selon une forme collective, et dans ce cas le «et » n'est pas une représentation, mais une donnée : la relation entre les chaises n'est pas un lien psychique qui viendrait après la donnée intuitive des chaises singulières, dans la mesure où elle est ellemême une donnée intuitive ${ }^{25}$. Il s'agit, contre le psychologisme, de récuser l'idée que la relation soit produite par la conscience réflexive, par exemple par un travail d'abstraction à partir des données sensibles. En effet, la relation ne peut pas se trouver réduite au statut d'un objet mental,

\footnotetext{
${ }^{21}$ Critique de la raison pure, Esthétique transcendantale : « Nous ne connaissons rien de ces objets que la manière dont nous les percevons, manière qui nous est propre, et peut fort bien n'être pas nécessaire pour tous les êtres, bien qu'elle le soit pour tout homme » (A42/B59), p. 801.

${ }^{22}$ Voir par exemple Herman Jean de Vleeschauwer, La déduction transcendantale dans l'œuvre de Kant, Anvers, Paris, La Haye, De Sikkel, Leroux, Nijhoff, 1934-1937, volume 1, p. 222.

${ }^{23}$ Voir les Prolégomènes à toute métaphysique future, $\$ 28$.

${ }^{24}$ Recherches logiques VI, § 51, p. 689 ; trad. p. 194-195.

${ }^{25}$ Voir Recherches logiques VI, § 56.
} 
mais elle doit être comprise comme un objet idéal visé par la conscience. Bien évidemment il y a quelque chose d'étonnant à parler d'une intuition de la relation et d'une donnée de la catégorie de la relation. Toute la difficulté est bien alors de concevoir comment cette catégorie devient accessible à partir de l'expérience sensible sans être sensible et sans être produite par la conscience ${ }^{26}$. Le projet de Husserl est bien de parvenir à délier la catégorie de la relation des facultés de représentation d'un sujet fini afin de restituer toute sa force à cette catégorie décisive. Sur ce point le nombre est un fil conducteur privilégié de l'analyse, puisqu'il n'est pas concevable d'avoir l'intuition catégoriale de nombre de façon isolée ${ }^{27}$ et qu'elle reconduit du domaine arithmétique qui est le sien : «L'intuition catégoriale est saisie de relations avant d'être donation d'objets ${ }^{28} \gg$.

Husserl peut alors élargir la thèse qui est la sienne : « $\mathrm{Si}$, sur une telle base, se produit l'intuition d'une relation, par exemple d'une relation entre partie et tout, alors l'acte relationnel a, lui aussi, le caractère d'évidence ${ }^{29} \gg$. La relation appartient bien en propre à l'objet et ne relève pas d'une opération secondaire d'abstraction, et en cela il n'est plus possible de séparer la vision de l'œil de l'inspection de l'esprit, puisque l'identité d'une chose n'est pas l'œuvre d'un esprit qui la conçoit, mais est bien une synthèse catégoriale se donnant dans une intuition. En conséquence, dans l'intuition catégoriale la relation est bien un nouvel objet de l'expérience, puisque la catégorie est constituée par « toutes les formes objectives qui sont issues des formes de l'appréhension, et non par les matériaux de l'appréhension ${ }^{30} »$. Dans cette rupture radicale envers l'ontologie traditionnelle, la relation est une forme de la proto-objectivité qui n'est pas un genre suprême par rapport à ses espèces. En effet, la relation n'est ni un concept purement sensible, ni un concept sensible mêlé à une forme catégoriale, mais est un concept purement catégoriel (syntaxique (et) ou ontologique (ensemble)) totalement indépendant de la particularité. De ce point de vue, la relation n'est pas intelligible à partir de la donation de l'objet sensible et est une forme de validité du sens. Si la relation est inséparable de ce qu'elle relie, il n'en demeure pas moins qu'en tant qu'elle constitue l'objectivité, elle est ce à quoi le sensualisme et le psychologisme demeurent aveugles. Certes la philosophie critique de Kant est une étape décisive, car elle libère la catégorie de relation du sensualisme, mais elle l'affaiblit en retour en ne pouvant pas la comprendre comme appartenant aux choses mêmes.

\section{La relation comme forme immanente du perçu}

Avec Husserl, on a donc pu libérer l'enquête sur la relation de toute forme d'empirisme, sans pour autant subjectiviser la relation, et cela en élucidant la nature de la perception catégoriale qui vise un nouvel objet. La relation n'a pas de teneur matériale et a seulement une teneur formelle ; elle n'a pas de lien direct avec une sphère ontique particulière. L'interrogation sur la relation a donc profondément changé, car il n'est plus question de savoir d'où vient la

\footnotetext{
${ }^{26}$ Dominique Pradelle souligne ici une difficulté : «Les textes essentiels de la sixième Recherche sont à ce titre décevants, car Husserl y démontre l'existence de l'intuition catégoriale par un raisonnement indirect, analogique et présomptif, sans mettre en évidence les structures noétiques de l'acte même ", "Qu'est-ce qu'une intuition catégoriale de nombre? », dans Marion Jean-Luc, Benoist Jocelyn, Courtine Jean-François directeurs, Husserl Edmund La représentation vide, Paris, PUF, 2003, p. 167.

${ }^{27}$ Voir Pradelle Dominique «Qu'est-ce qu'une intuition catégoriale de nombre ?», p. 172.

${ }^{28}$ «Qu'est-ce qu'une intuition catégoriale de nombre?», p. 180.

29 «Findet nun auf solcher Grundlage die Anschauung einer Beziehung statt, z.B. einer Beziehung zwischen Teil und Ganzem, so hat auch der bezienhende Akt den Charakter der Evidenz », Recherches logiques VI, § 57, p. 704; trad. p. 212.

${ }^{30}$ Recherches logiques VI, § 58, p. 709 ; trad. p. 217.
} 
catégorie de la relation, quelle serait son origine subjective, mais il s'agit cette fois d'élucider quels sont les actes noétiques rendant possible une intuition catégoriale de la relation. Husserl écrit :

Si par exemple une intuition de la relation se trouve à la base de l'abstraction, il se peut que la conscience abstractive se dirige sur la forme relationnelle in specie, de telle manière que tout l'aspect sensible des fondements relationnels demeure hors-jeu ${ }^{31}$.

Ainsi la catégorie de la relation ne se trouve pas saisie à partir d'une intuition simple liée à l'objet individuel, ni par un acte d'abstraction sensible permettant la saisie d'un objet général, ni par un acte d'idéalisation, mais on y accède par cet acte $d$ ' « abstraction » purement catégorial qu'est l'acte formalisant. C'est donc bien l'essence de l'objet qui détermine le phénomène de la relation et non la nature du sujet connaissant; la relation n'est pas une catégorie à part, qui serait à reconduire à des catégories plus importantes comme la substance ou la qualité, car elle n'est rien d'extérieur à l'objet, mais est donnée avec l'objet lui-même. Plus encore, la réduction phénoménologique met en évidence que la relation est elle-même un objet qui se donne à la conscience. Une telle relation est indépendante de toute subjectivité actuelle et la catégorie de relation est un concept structurel originairement perçu. Cela confirme à quel point Husserl s'éloigne de la compréhension de la relation selon la hiérarchie aristotélicienne et montre comment cette catégorie relève de l'ontologie formelle, en tant que catégorie pure qui ne vient pas de l'expérience interne, mais de la donnée des $\operatorname{choses}^{32}$. Husserl est donc parvenu à mettre en lumière que la relation n'est ni une construction intellectuelle, ni ce qui relève d'un préjugé empiriste.

A partir de Husserl, la conception aristotélicienne de la relation comme détermination accidentelle extérieure à l'essence se trouve radicalement renversée et la catégorie de la relation reprend une importance tout à fait considérable, dans la mesure où voir, c'est toujours voir une relation, puisqu'un objet ne se donne jamais seul, mais toujours sur l'horizon d'un univers unitaire (einheitlichen Universum), comme Husserl le dira dans le $\S 15$ des Méditations cartésiennes. La réflexion transcendantale mettra en lumière la relation comme une condition nécessaire de tout apparaître d'un objet, puisqu'il y a un a priori de corrélation entre la conscience de cet univers et la conscience de l'objet. Dès lors, selon le $\S 18$ des Méditations cartésiennes, la conscience d'une relation conduit à élucider comment se constitue « syntaxiquement » le cogitatum, dans la passivité et l'activité. La relation fait donc partie des catégories formelles et logiques qui ne sont pas issues sur les formes catégoriales du jugement, mais qui sont déduites transcendentalement à partir du fil conducteur de l'objet qui devient guide transcendantal. Autrement dit, la relation montre la chose même et pas uniquement les circonstances de sa manifestation, et c'est cela qui n'avait jamais pu être vraiment pensé d'Aristote à Kant. Ce sont alors toutes les distinctions traditionnelles qui s'effacent avec l'intuition catégoriale de la relation. Néanmoins cela conduit Husserl à tenir un équilibre délicat, puisqu'il ne peut pas y avoir d'origine empirique de la catégorie de la relation et que, pourtant, l'intuition de la relation doit être fondée sur la donation d'objets sensibles ${ }^{33}$. Husserl parvientil à élucider la manière dont l'intuition de la relation demeure fondée sur la donation sensible, si elle demeure comprise comme la saisie d'un nouvel objet? Est-il possible de totalement

\footnotetext{
${ }^{31}$ Recherches logiques VI, § 60, p. 713 ; trad. p. 221.

${ }^{32}$ Sur ce point voir le $\S 10$ des Idées directrices pour une phénoménologie et une philosophie phénoménologique pures, livre I.

${ }^{33}$ Voir sur ce point Richard Cobb-Stevens, Husserl et la philosophie analytique, trad. E. Paquette, Paris, Vrin, 1998, notamment p. 193.
} 
réduire le fait que la relation soit une production de l'esprit humain ? Husserl a bien tenté de montrer que l'intuition catégoriale de la relation ouvre un accès à la rationalité du monde, sans que celle-ci soit considérée comme relative à la subjectivité. En refusant le passage kantien des conditions de possibilité de l'expérience à la possibilité des objets de l'expérience, Husserl parvient à mettre en lumière que la relation n'est pas une forme pure de l'acte d'intuitionner et qu'elle doit être considérée comme une forme de ce qui est intuitionné en tant que tel. En effet, la force des analyses de Husserl, en élucidant la relation comme une structure immanente du perçu, est de libérer la catégorie de la relation de toute dimension anthropologique, pour décrire métaphysiquement la relation au-delà de toute considération empirique. Dès lors, l'intuition catégoriale de la relation ne correspond pas à une plus haute possibilité du sujet, mais est bien l'accès à une plus haute couche de sens de l'objet, accès donnant à voir la rationalité du monde. Ainsi, en mettant en évidence que ce sont les choses mêmes qui prescrivent à tout sujet concevable l'intuition de la relation, Husserl introduit un tout autre concept de monde. Néanmoins les difficultés rencontrées pour élucider les actes noétiques propres à la relation montrent que Husserl comprend toujours la relation comme une catégorie, c'est-dire comme une détermination de ce qui est devant la main, disponible, sans effectuer le pas qui consiste à la comprendre comme un existential. En effet, pour comprendre que la relation est quelque chose de l'être, il est nécessaire de ne plus faire de l'identité un trait de l'être en s'ouvrant à l'idée que chaque chose séjourne en rassemblant : la relation dans sa signification verbale serait alors la façon dont chaque chose fait monde en séjournant avec les autres choses. En montrant que la relation n'est pas produite par l'esprit depuis une intériorité qui s'oppose à une extériorité et qu'elle est au contraire une objectité fondée, Husserl a conduit, sans le prévoir, à une tout autre compréhension de la relation.

\section{Conclusion}

On a ainsi voulu montrer en quoi l'intuition catégoriale de la relation dans la phénoménologie de Husserl est un tournant, mais un tournant très paradoxal, car d'un côté Husserl rompt avec la conception traditionnelle de la relation en montrant qu'elle peut aussi être considérée comme un trait de l'être d'un point de vue constitutif et, d'un autre côté, la phénoménologie après Husserl voudra remonter à une relation plus originaire que la relation catégoriale qui bien que non sensible est intuitionnée comme fondée dans le sensible. Husserl instaure donc une rupture en restituant à la relation son singulier et en lui redonnant une consistance ontologique ${ }^{34}$ en montrant qu'elle ne saurait être indépendante d'intuitions sensibles donatrices, mais après lui une partie de la phénoménologie ouvrira d'autres voies en cherchant à élucider une relation qui ne soit plus une corrélation entre étants. En désubjectivisant la relation, Husserl a ouvert, bien malgré lui et sans le savoir ni le vouloir, à une autre compréhension de la relation dans laquelle elle n'est plus reconduite aux actes subjectifs qui la rendent possible. On a déjà évoqué Heidegger (le Mitsein) et Merleau-Ponty (la réversibilité) ou la «nostrité » de Binswanger, mais il est également possible de penser aux analyses contemporaines de Scheler sur la personne commune, qui est bien autre chose que la somme de subjectivités singulières, dans la mesure où elle est l'évidence d'une

\footnotetext{
${ }^{34}$ Même si cela soulève plusieurs difficultés, voir Richard Cobb-Stevens, Husserl et la philosophie analytique, op. cit., p. 191 et sv.
} 
coresponsabilité. La relation éthique n'est pas ce qui vient s'ajouter à la subjectivité singulière, mais elle est ce qui lui appartient de manière originaire ${ }^{35}$.

Cela dit, au-delà encore de Scheler, ou de la conception heideggérienne du Mitsein, il est possible d'envisager la profonde rupture instaurée par Levinas vis-à-vis de la conception husserlienne de la relation toujours reconduite à la relation de la noèse et du noème. Dans les textes de Levinas il ne s'agit plus d'une relation transcendantale telle que Husserl a pu la mettre en évidence, mais d'une « relation qui rattache le Moi à Autrui - idée de 1'Infini ${ }^{36}$ » qui ne relève plus d'une perspective gnoséologique. Sans pouvoir développer, il suffit d'indiquer à titre de confrontation que la relation morale n'est plus ici catégoriale, qu'elle n'est plus issue de la réflexion, et c'est pourquoi Levinas n'écrit pas une éthique et ne cherche pas à fonder la morale. Son intention est au contraire de décrire un «lien anarchique ${ }^{37} »$, une relation de face à face qui n'est ni celle de la constitution de l'alter ego, ni celle du Mitsein. Il n'est donc plus question de relation catégoriale, car « la relation avec l'autre est la relation avec un mystère ${ }^{38}$ » sur laquelle ne peut tomber la clarté de l'évidence : «Relation avec l'autre qui ne sera jamais le fait de saisir une possibilité. Il faudrait la caractériser en des termes qui tranchent sur les relations qui décrivent la lumière ${ }^{39} \gg$. La relation ici n'est plus de l'ordre de l'expérience, de la synthèse, car «c'est la relation avec l'altérité, avec le mystère, c'est-à-dire avec l'avenir ${ }^{40}$ ». Si Husserl a rompu avec la conception kantienne de la relation, Levinas rompt avec Husserl en pensant une relation qui n'est plus de l'ordre de la synthèse. Cette relation exceptionnelle (audelà de l'alternative relation accidentelle, relation essentielle) n'est plus ce qui fait l'objet d'une intuition catégoriale et renvoie à des situations concrètes d'existence dans lesquelles l'homme est toujours déjà pris. Il y a une paternité plus originaire que celle, catégoriale, dans laquelle le père peut tenter de se mettre à la place de son fils. Levinas restaure donc bien l'idée platonicienne qu'au sein même de l'être il puisse y avoir une altérité absolue contre la thèse husserlienne qui réduirait trop la relation à la donnée d'une identité. Autrement dit, si on ne veut pas réduire la relation à une réalité nominale, il ne suffit pas de mettre en évidence que l'être-ensemble appartient à la donnée, mais il convient de restituer sa consistance ontologique à l'altérité en défendant la thèse qu'il n'y a pas d'être absolu, même sous la figure de l'ego transcendantal, qui serait le non-relatif et le fondement de tous les relatifs. Pour Levinas il s'agit d'aller encore plus loin que toute philosophie idéaliste qui manque la relation dans la mesure où elle pose le primat de la pensée sur l'existence. Il reconnaît à Heidegger de lui avoir appris la tâche de ne pas séparer philosopher et vivre, néanmoins le projet de Levinas est de défendre philosophiquement, et non pas théologiquement, que la relation des relations est celle du fini à l'infini, que la relation éthique comme expérience de l'Autre qui n'abolit pas la séparation est l'origine du sens, c'est-à-dire aussi celle du « et » ainsi que de «l'être-ensemble ». Tel est le paradoxe historique dont il s'agit de prendre la mesure : au moment même où dans l'histoire de la métaphysique les relations ne sont plus réduites à des accidents inhérents aux substances, le principe d'identité lui-même finit par être remis en cause, comme si faire apparaître la relation

\footnotetext{
${ }^{35}$ Voir Le Formalisme en éthique et l'éthique matériale des valeurs, Elibron Classics, 2007, facsimilé de l'édition Niemeyer de 1921, p. 546 ; trad. Maurice de Gandillac, Paris, Gallimard, 1955, p. 524. Je me permets de renvoyer à mon étude: «Personne commune et coresponsabilité selon Scheler», dans Max Scheler Ethique et phénoménologie, dir. Gabriel Mahéo et Emmanuel Housset, Presses Universitaires de Rennes, 2015, p. 35-60.

${ }^{36}$ Humanisme de l'autre homme, Le livre de poche, Paris, Fata Morgana, 1972, p. 54.

${ }^{37}$ Humanisme de l'autre homme, p. 88.

${ }^{38}$ Le temps et l'autre, Paris, PUF, $5^{\text {ème }}$ édition Quadrige, 1994, p. 63.

${ }^{39}$ Le temps et l'autre, p. 64.

${ }^{40}$ Le temps et l'autre, p. 81.
} 
comme phénomène par la réduction phénoménologique avait conduit la phénoménologie à ce qui lie l'être et le temps. Sans vouloir remettre en cause l'importance des diverses pensées d'une relation originaire, préréflexive, on peut tout de même se demander s'il peut y avoir un langage de cette relation originaire. Ne doit-elle pas se dire dans le langage du monde, dans celui de la catégorie ? Cela confirmerait l'idée que cette relation originaire échappe par principe au pur regard de l'essence et qu'elle n'est saisie qu'indirectement comme ce qui maintient vivante l'intuition catégoriale de la relation. Dès lors il n'est pas question d'abolir la relation comme catégorie, même si c'est finalement une relation originaire qui «donne » à la relation catégoriale d'être la « donnée » elle-même et non une simple synthèse extérieure. Si l'intuition catégoriale de la relation donne à voir la rationalité du monde, la relation au mystère montre que la raison ne peut jamais se retourner pleinement sur elle-même pour se clore en un système, en une totalité. Quoi qu'il en soit, on peut maintenant avancer la thèse suivante : En permettant de dépasser l'opposition de la substance et de l'accident, en ne considérant plus la relation comme une catégorie dérivée, en montrant que la relation à une altérité appartient à la donnée elle-même et qu'il appartient à l'être d'une chose de se rapporter à autre chose qu'elle, Husserl a ouvert la voie, sans le vouloir, aux pensées de la subjectivité exposée, voire brisée.

Le but de cette étude n'était pas d'exposer toute la question de la relation dans la phénoménologie de Husserl, ni d'effectuer une confrontation effective avec d'autres compréhension de la relation après Husserl, mais de tenter de mettre en lumière dans quelle mesure l'intuition de la relation telle qu'elle est exposée dans les Recherches logiques marque tout de même une rupture historique avec Aristote, Locke et Kant, et anime la réflexion de Husserl sur la conscience du temps, la constitution de l'objet, l'expérience d'autrui et même celle de la communauté. Il s'agissait également de souligner en quoi Husserl était conscient des difficultés propres à cette thèse d'une dimension intuitive de la relation et à quel point il est complexe de conduire cette intuition de la relation à la clarté de l'évidence dans une réflexion transcendantale. Mais bien sûr au-delà de ces trois premières intentions le projet était également d'établir qu'il y a là une œuvre de percée et que la rupture effectuée par Husserl avec une conception empiriste, comme avec une conception idéaliste, de la relation en accordant une place essentielle à la sensibilité dans la conception de la connaissance est ce qui va rendre possible une autre rupture : élucider une relation originaire à l'infini, à l'incompréhensible, comme origine du sens. La force d'une grande philosophie est ainsi d'ouvrir des chemins qui vont au-delà d'elle. 